\title{
Adherence to response-guided pegylated interferon and ribavirin for people who inject drugs with hepatitis $C$ virus genotype 2/3 infection: the ACTIVATE study
}

Evan B. Cunningham ${ }^{1 *}$ (D), Behzad Hajarizadeh ${ }^{1}$, Olav Dalgard², Janaki Amin ${ }^{1}$, Margaret Hellard ${ }^{14}$, Graham R Foster ${ }^{3}$, Philip Bruggmann ${ }^{4}$, Brian Conway ${ }^{5}$, Markus Backmund ${ }^{6}$, Geert Robaeys ${ }^{7,8,9}$, Tracy Swan ${ }^{10}$, Philippa S. Marks ${ }^{1}$, Sophie Quiene ${ }^{1}$, Tanya L Applegate ${ }^{1}$, Martin Weltman ${ }^{11}$, David Shaw ${ }^{12}$, Adrian Dunlop ${ }^{13}$, Julie Bruneau ${ }^{15}$, Håvard Midgard ${ }^{2}$, Stefan Bourgeois ${ }^{16}$, Maria Christine Thurnheer ${ }^{17}$, Gregory J Dore ${ }^{1}$, Jason Grebely ${ }^{1}$ and on behalf of the ACTIVATE Study Group

\begin{abstract}
Background: The aims of this analysis were to investigate treatment completion and adherence among people with ongoing injecting drug use or receiving opioid substitution therapy (OST) in a study of response-guided therapy for chronic HCV genotypes 2/3 infection.

Methods: ACTIVATE was a multicenter clinical trial recruited between 2012 and 2014. Participants with genotypes $2 / 3$ were treated with directly observed peg-interferon alfa-2b (PEG-IFN) and self-administered ribavirin for 12 (undetectable HCV RNA at week 4) or 24 weeks (detectable HCV RNA at week 4). Outcomes included treatment completion, PEG-IFN adherence, ribavirin adherence, and sustained virological response (SVR, undetectable HCV RNA > 12 weeks post-treatment).

Results: Among 93 people treated, 59\% had recently injected drugs (past month), 77\% were receiving OST and $56 \%$ injected drugs during therapy. Overall, 76\% completed treatment. Mean on-treatment adherence to PEG-IFN and ribavirin were $98.2 \%$ and $94.6 \%$. Overall, $6 \%$ of participants missed $>1$ dose of PEG-IFN and $31 \%$ took $<95 \%$ of their prescribed ribavirin., Higher treatment completion was observed among those receiving 12 vs. 24 weeks of treatment ( $97 \%$ vs. $46 \%, P<0.001$ ) while the proportion of participants with $95 \%$ on-treatment ribavirin adherence was similar between groups (67\% vs. $72 \%, P=0.664)$. Receiving 12 weeks of therapy was independently associated with treatment completion. No factors were associated with 95\% RBV adherence. Neither recent injecting drug use at baseline nor during therapy was associated with treatment completion or adherence to ribavirin. In adjusted analysis, treatment completion was associated with SVR (aOR 23.9, 95\% Cl 2.9-193.8).
\end{abstract}

Conclusions: This study demonstrated a high adherence to directly observed PEG-IFN and self-administered ribavirin among people with ongoing injecting drug use or receiving OST. These data also suggest that shortening therapy from 24 to 12 weeks can lead to improved treatment completion. Treatment completion was associated with improved response to therapy. ACTIVATE trial registration number: NCT01364090 - May 31, 2011

Keywords: Hepatitis C, Treatment, PWID, Injection drug use, Adherence

\footnotetext{
* Correspondence: ecunningham@Kirby.Unsw.Edu.Au

${ }^{1}$ The Kirby Institute, UNSW Sydney, Sydney, NSW, Australia

Full list of author information is available at the end of the article
} 


\section{Background}

Among people who inject drugs (PWID), there is a substantial burden of hepatitis $\mathrm{C}$ virus $(\mathrm{HCV})$ infection $[1,2]$. Adherence [3-6] and treatment completion [3] are associated with sustained virologic response (SVR). Adherence to $\mathrm{HCV}$ therapy among PWID is of particular interest given the high cost of new direct-acting antiviral (DAA) therapies and the importance of maximizing the chance of successful therapy.

Adherence refers to the extent to which a person's behaviour, with respect to timing, dosage and frequency of taking medication, corresponds with agreed recommendations from a healthcare provider [7, 8]. Medication adherence research has been performed in a variety of medical conditions, including diabetes, hypertension, arthritis, pulmonary diseases, HIV and others [9] and on average, patients take $79 \%$ of prescribed doses of medications [7]. Adherence to $\mathrm{HCV}$ therapy is often defined measuring " $80 / 80$ adherence", or the receipt of $\geq 80 \%$ of scheduled doses for $\geq 80 \%$ of the scheduled treatment period [7]. However, this definition combines the two distinct concepts of treatment completion and missed doses during therapy. Understanding the two concepts individually is important for understanding adherence in this population of PWID.

Among health practitioners, HCV therapy is sometimes withheld from people with ongoing injecting drug use, based on concerns of poor adherence to therapy, and risk of reinfection [10]. However, there are few studies that have evaluated adherence to $\mathrm{HCV}$ therapy among people with ongoing injecting drug use. Understanding adherence to therapy among PWID is a key component to the scale-up of DAA therapy among this population.

ACTIVATE is a multicentre international trial evaluating the efficacy of response-guided directly observed pegylated-interferon and self-administered ribavirin therapy for chronic HCV genotypes $2 / 3$ infection among people with ongoing injecting drug use or receiving opioid substitution therapy (OST). Participants with genotypes 2/ 3 were treated with directly observed peg-interferon alfa-2b and self-administered ribavirin for either 12 weeks (in those with undetectable HCV RNA at week 4, RVR) or 24 weeks (in those with detectable HCV RNA at week 4, no RVR). The primary analysis from this study demonstrated that cirrhosis (vs. no/mild fibrosis [adjusted odds ratio (aOR) 0.33 , 95\% CI 0.13, 0.86]) predicted reduced SVR, while response at week 4 was associated with increased SVR (aOR 8.11, 95\% CI 2.73, 24.10) [11]. While the PEG-IFN based regimen used in this study has been replaced in many settings with new DAA therapies, data on adherence to HCV therapy among PWID is still needed. Given the increased tolerability and simplicity of new DAA regimens compared to PEG-IFN based therapies, results in this study likely represent a lower bound of adherence among PWID using new DAA therapies.

The primary aim of this analysis was to evaluate treatment completion, adherence to therapy and associated factors (including impact of treatment duration) in the ACTIVATE study. Further, this analysis also investigated the effect of treatment completion and on-treatment adherence on response to HCV therapy (as measured by SVR).

\section{Methods \\ Study participants}

From May 11 2012, to September 30 2014, participants were enrolled at 17 sites in Australia $(n=5)$, Belgium $(n=2)$, Canada $(n=3)$, Germany $(n=1)$, Norway $(n=2)$, Switzerland $(n=3)$ and the United Kingdom $(n=1)$. The last participant visit was July 152015 . Study recruitment was conducted through a network of drug and alcohol clinics $(n=3)$, office-based practices $(n=2)$, hospital clinics $(n=9)$, and community clinics $(n=3)$.

Participants had to be more than 18 years of age, have chronic $\mathrm{HCV}$ genotype 2 or 3 infection, be $\mathrm{HCV}$ treatment-naïe, and have reported recent injecting drug use, defined as injecting drug use within 12 weeks of enrolment. Due to slower than anticipated recruitment, on June 26 2013, a study protocol amendment was implemented to also include people currently receiving OST with no recent injection drug use and people who had injected within 24 weeks prior to enrolment. Participants with HIV infection and decompensated liver disease were excluded. Full eligibility has been previously published [11].

\section{Study design and intervention}

ACTIVATE was an international, multicentre openlabel study. Participants received directly observed pegylated interferon alfa-2b (PEG-IFN, $1.5 \mu \mathrm{g} / \mathrm{kg} /$ week) and self-administered ribavirin (RBV, 800-1400 mg daily, weight-based).

Participants with a rapid virological response [RVR, defined as non-quantifiable HCV RNA $(<15 \mathrm{IU} / \mathrm{ml}$ detected and $<15 \mathrm{IU} / \mathrm{ml}$ undetected) or undetectable HCV RNA on qualitative assay at week 4] received 12 weeks of therapy (shortened duration). Participants without an RVR [defined as quantifiable HCV RNA ( $\geq 15 \mathrm{IU} / \mathrm{ml})$ or detectable HCV RNA on qualitative assay at week 4] received 24 weeks of therapy (standard duration).

\section{Study assessments}

Screening assessments included serum HCV RNA levels, HCV genotype, standard laboratory and clinical testing and self-reported behavioural questionnaires (details have been previously reported [11]).

HCV RNA levels and HCV genotype and subtype were measured as previously described [11]. HCV RNA testing 
was performed on samples collected at screening, baseline, and weeks 4, 12, 24, 36 and 48 (standard duration). All adverse events were recorded and graded according to a standard scale (details have been previously reported [11]).

Directly observed PEG-IFN adherence was recorded by the study nurse. RBV adherence was determined through the return of unused RBV pills. Self-reported RBV adherence was also measured monthly during study visits while on treatment by a patient-administered questionnaire and was used where returned pill counts were unavailable for that time point.

All participants completed a self-administered questionnaire at enrolment (pre-treatment assessment), at baseline (treatment commencement), every 4th week during treatment, and at 12 and 24 weeks of follow-up. The questionnaires collected information on demographics (age, gender, ethnicity, education level, housing status and history of imprisonment), drug and alcohol use, injecting risk behaviours (injection frequency, use of non-sterile needles, needle and syringe borrowing or lending, and injecting paraphernalia [spoons or mixing containers, drug solution/mix, water or filter] sharing), drug treatment, and symptoms of psychological distress (Depression Anxiety Stress Scale, DASS-21). Stable housing was defined as living in a rented or privately owned house or flat..

Social functioning was measured using the short-form Opioid Treatment Index Social Functioning Scale [12]. Social functioning are scored as a sum of the coded responses with higher scores indicating lower social functioning. Alcohol consumption was evaluated by the

Table 1 Baseline demographic and clinical characteristics stratified by 12 week and 24 week duration $(n=93)$

\begin{tabular}{|c|c|c|c|}
\hline Characteristic, n (\%) & Overall $(n=93)$ & 12 week $(n=61)$ & 24 week $(n=26)$ \\
\hline Age, median $(25 \%, 75 \%)$ & $41(35-49)$ & $41(34-49)$ & $40(35-48)$ \\
\hline Male sex, n (\%) & $77(83)$ & $49(80)$ & $23(88)$ \\
\hline $\begin{array}{l}\text { Drug use in the last } 6 \text { months } \\
\text { (injecting/non-injecting) }\end{array}$ & $77(83)$ & $48(79)$ & $25(96)$ \\
\hline Injecting drug use in the last month & $55(59)$ & $39(64)$ & $15(58)$ \\
\hline Heroin & $33(35)$ & $23(37)$ & $10(38)$ \\
\hline Cocaine & $10(11)$ & $7(11)$ & $3(12)$ \\
\hline Amphetamines & $14(15)$ & $7(11)$ & $6(23)$ \\
\hline Other opiates & $11(12)$ & $8(13)$ & $3(12)$ \\
\hline Benzodiazapines & $2(2)$ & $2(3)$ & $0(0)$ \\
\hline \multicolumn{4}{|c|}{ Injecting drug use frequency in the last month } \\
\hline Never & $38(41)$ & $22(36)$ & $11(42)$ \\
\hline$<$ daily & $40(43)$ & $29(48)$ & $10(38)$ \\
\hline$>$ daily & $15(16)$ & $10(16)$ & $5(19)$ \\
\hline Opioid substitution treatment (ever) & $82(88)$ & $56(92)$ & $21(81)$ \\
\hline \multicolumn{4}{|c|}{ OST and recent injecting (past month) at enrolment } \\
\hline No OST, recent injecting & $30(32)$ & $20(33)$ & $9(33)$ \\
\hline OST, no recent injecting & $23(25)$ & $15(25)$ & $5(19)$ \\
\hline OST, recent injecting & $40(43)$ & $26(43)$ & $12(44)$ \\
\hline \multicolumn{4}{|c|}{ OST and recent injecting (past month) at baseline } \\
\hline No OST, recent injecting & $21(23)$ & $14(23)$ & $6(23)$ \\
\hline OST, no recent injecting & $34(37)$ & $21(34)$ & $8(31)$ \\
\hline OST, recent injecting & $38(41)$ & $26(43)$ & $12(46)$ \\
\hline \multicolumn{4}{|l|}{ Stage of liver disease } \\
\hline No or mild fibrosis (F0-F1) & $63(68)$ & $44(72)$ & $16(62)$ \\
\hline Moderate or advanced fibrosis (F2-F3) & $20(22)$ & $12(20)$ & $5(19)$ \\
\hline Cirrhosis (F4) & $10(11)$ & $5(8)$ & $5(19)$ \\
\hline \multicolumn{4}{|l|}{ Study site distribution } \\
\hline Europe & $38(41)$ & $24(39)$ & $13(50)$ \\
\hline Australia & $40(43)$ & $27(44)$ & $9(35)$ \\
\hline Canada & $15(16)$ & $10(16)$ & $4(15)$ \\
\hline
\end{tabular}


Alcohol Use Disorders Identification Test-Consumption (AUDIT-C), derived from the first three questions of the full AUDIT (scores $\geq 3$ and $\geq 4$ indicate hazardous consumption or active alcohol use disorders among women and men, respectively) [13]. Depression was measured using DASS 21 with a depression score of $\geq 10$ indicating depression.

\section{Study definitions \\ Treatment completion}

Participants with no early discontinuation of PEG-IFN/ RBV therapy prior to the per-protocol planned end of treatment (12 or 24 weeks for shortened or standard therapy respectively) were defined as having completed treatment. Participants were deemed to have discontinued treatment early if for any reason (e.g. physician advised treatment discontinuation, virological non-response, lost to follow up, patient decision, etc.) a participant did not reach the per protocol defined end of treatment.

\section{On-treatment adherence}

On-treatment adherence was calculated by determining the number of doses taken as proportion of the expected number of doses during the time on treatment. This measures the proportion of doses received from the time that treatment was initiated until treatment was discontinued or completed.

\section{Dose modification}

A physician directed increase or reduction in the dose at any time during treatment.

\section{Study outcomes}

The main study outcomes were to assess treatment completion, and on-treatment PEG-IFN and RBV adherence. Evaluation of adherence was based on all participants who received at least one injection of PEG-IFN. Treatment success was defined as undetectable qualitative HCV RNA rates at week 12 (SVR).

\section{Statistical analysis}

Treatment completion and $95 \%$ on-treatment adherence (at least 95\% of scheduled doses were taken) were assessed. Bi-variate comparisons of characteristics of participants and different measures of adherence across treatment arms were tested using the chi-squared test or Fisher's exact test as appropriate. Time to treatment discontinuation was evaluated using Kaplan Meier analysis. The impact of treatment completion and on-treatment adherence (both PEG-IFN and ribavirin) on SVR were also evaluated.

Logistic regression analyses were used to estimate crude and adjusted odds ratios (OR) and corresponding 95\% confidence intervals $(95 \% \mathrm{CI})$ to identify predictors of
$\mathrm{HCV}$ treatment completion and on-treatment ribavirin adherence (at least $95 \%$ of scheduled doses were taken). In unadjusted analyses, potential predictors were determined a priori and included sex, age, education, accommodation, employment, current OST treatment, social functioning, current depression, alcohol consumption, injection drug use at baseline (past month), injecting behaviours (frequency and drug injected), and treatment arm. Social functioning was calculated using a validated scale from

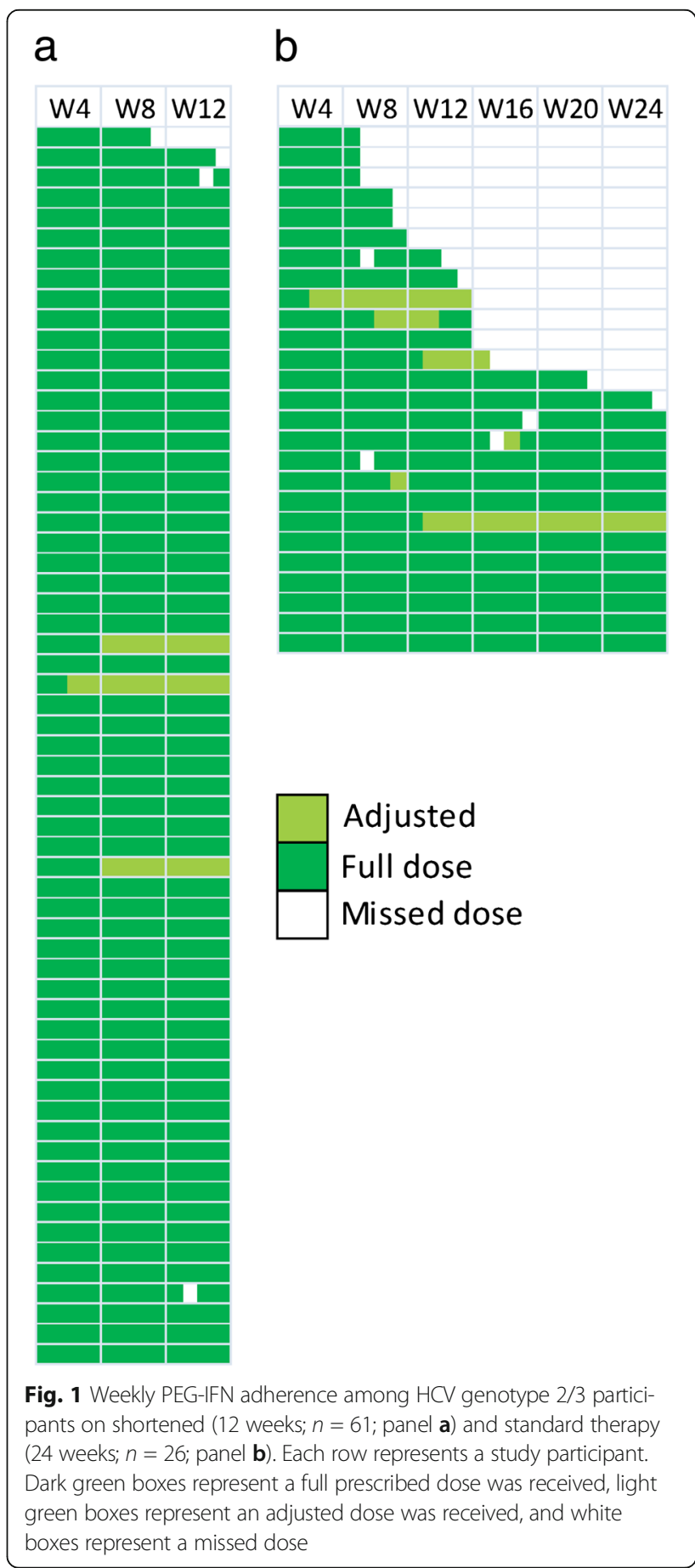


Table 2 Adherence to PEG-IFN and RBV among the overall population, those on 12 weeks of therapy, and those on 24 weeks of therapy

\begin{tabular}{|c|c|c|c|}
\hline Variable & Overall $(n=93)$ & 12 week $(n=61)$ & 24 week $(n=26)$ \\
\hline Treatment completion & $71(76)$ & $59(97)$ & $12(46)$ \\
\hline Mean on-treatment PEG-IFN adherence percent, (SD) & $98.2(4.5)$ & $98.5(3.1)$ & $98.7(3.0)$ \\
\hline Mean on-treatment ribavirin adherence percent, (SD) & $94.6(8.8)$ & $94.8(8.1)$ & $94.1(10.8)$ \\
\hline \multicolumn{4}{|l|}{ Missed doses of PEG-IFN, $n(\%)$} \\
\hline No missed doses & $87(94)$ & $59(97)$ & $22(85)$ \\
\hline 1 missed dose & $6(6)$ & $2(3)$ & $4(15)$ \\
\hline 2-5 missed doses & $0(0)$ & $0(0)$ & $0(0)$ \\
\hline$>5$ missed doses & $0(0)$ & $0(0)$ & $0(0)$ \\
\hline \multicolumn{4}{|l|}{ Missed doses of ribavirin, $\mathrm{n}(\%)$} \\
\hline $100 \%$ of doses taken & $22(25)$ & $16(26)$ & $5(20)$ \\
\hline $95 \%-<100 \%$ of doses taken & $38(44)$ & $25(41)$ & $13(52)$ \\
\hline $90 \%-<95 \%$ of doses taken & $15(17)$ & $11(18)$ & $4(16)$ \\
\hline $80 \%-<90 \%$ of doses taken & $7(8)$ & $6(10)$ & $1(4)$ \\
\hline$<80 \%$ of doses taken & $5(6)$ & $3(5)$ & $2(8)$ \\
\hline \multicolumn{4}{|l|}{ Number of weeks of PEG-IFN therapy, n (\%) } \\
\hline 24 weeks & $12(13)$ & NA & $12(46)$ \\
\hline 13 to 23 weeks & $3(3)$ & NA & $3(3)$ \\
\hline 7 to 12 weeks & $69(74)$ & $61(100)$ & $8(31)$ \\
\hline 0 to 6 weeks & $9(10)$ & $0(0)$ & $3(12)$ \\
\hline \multicolumn{4}{|l|}{ Weeks on PEG-IFN therapy } \\
\hline Mean, n (SD) & NA & $11.9(0.65)$ & $16.8(7.8)$ \\
\hline Median, n (IQR) & NA & $12(12-12)$ & $21(10-24)$ \\
\hline PEG-IFN dose-modification & $9(10)$ & $3(5)$ & $6(23)$ \\
\hline Ribavirin dose-modification & $21(23)$ & $12(20)$ & $9(35)$ \\
\hline
\end{tabular}

the Opiate Treatment Index [27] that addresses employment, residential stability, and inter-personal conflict as well as social support. A higher score reflects poorer social functioning. This scale has been validated among opiate users in Australia (range, 0-48) [27]. All variables with $p<0.20$ in the bivariate analysis were considered for multivariate logistic regression models using a backward stepwise approach, sequentially eliminated and subject to the result of a likelihood ratio test. Statistically significant differences were assessed at $p<0.05 ; p$ values are twosided. Adjusted models for factors associated with SVR were adjusted for all variables found to be associated with SVR in the primary analysis (fibrosis stage and treatment group) as well as by study site using cluster-robust standard errors [11].

Finally, to determine whether later study visits were associated with RBV adherence, generalized estimating equation (GEE) methods were used. Unadjusted and adjusted GEE models were specified using a gaussian family function. Odds ratios (ORs) with corresponding 95\% confidence intervals (CIs) and $p$-values were

Fig. 2 Time to treatment discontinuation among study participants who were in the shortened (12 week; $n=61$ ) and standard (24 week; $n=26)$ arm 
statistical package Stata v13.1 (College Station, TX, United States).

\section{Results}

\section{Participant characteristics}

A full description of the participant characteristics is described in the primary paper [11]. In summary, 93 patients were enrolled in the study between May 2012 and August 2014 and initiated PEG-IFN/ribavirin therapy: median age $41,83 \%$ male, $77 \%$ on OST, $59 \%$ injecting in the past month (Table 1).

\section{PEG-IFN and RBV adherence and early treatment discontinuation}

Of the 93 participants who initiated therapy, six participants discontinued therapy before week 4 . Reasons for discontinuing therapy prior to week 4 include side effects $(n=3)$, unwillingness to continue treatment $(n=1)$, loss to follow up $(n=1)$, and imprisonment $(n=1)$. Among the remaining 87 participants, $70 \%$ $(n=61)$ were HCV RNA undetectable at week 4 (rapid virological response, RVR) and were scheduled to receive 12 weeks of therapy (shortened duration) and 30\% $(n=26)$ did not have an RVR at week 4 and were scheduled to receive 24 weeks of therapy (standard duration).

An abbreviated description of participant adherence is described in the primary paper [11]. Weekly PEG-IFN adherence among those receiving 12 weeks of therapy and 24 weeks of therapy is shown in Fig. 1. Among the entire study population, $76 \%(n=71)$ completed $\mathrm{HCV}$ therapy (Table 2). Time to discontinuation stratified by duration of therapy is shown in Fig. 2. The median time to discontinuation was 12 weeks and 23 weeks in the 12 week and 24 week groups respectively. Mean ontreatment adherence to PEG-IFN and ribavirin were 98\% and $95 \%$ (Table 2). Overall, $6 \%$ of participants missed $>1$ dose of PEG-IFN and 31\% missed $>5 \%$ of their doses of ribavirin.

Completion of treatment was more frequent in the 12 week group compared to the 24 week group ( $97 \%$ vs. $46 \%, P<0.001)$. In the 12 week group, completion of 0 $4,5-8$, and $9-12$ weeks of therapy was demonstrated by $0 \%(n=0), 2 \%(n=1)$, and $98 \%(n=60)$, respectively. In the 24 week arm, completion of $0-4,5-8,9-12,13-23$, and 24 weeks was demonstrated by $0 \%(n=0), 19 \%$ $(n=5), 23 \%(n=6), 3 \%(n=3)$ and $46 \%(n=12)$ respectively. Reasons for discontinuing prior to the planned end of therapy include side effects (overall, $n=11$; 12 week, $n=1$; 24 week, $n=7$ ), patient unwillingness (overall, $n=5$; 12 week, $n=1 ; 24$ week, $n=3$ ), patient lost to follow-up (overall, $n=4 ; 12$ week, $n=0$; 24 week, $n=3$ ), and virological failure (overall, $n=1$; 12 week, $n=0 ; 24$ week, $n=1)$. The mean number of weeks of PEG-IFN therapy was 11.9 and 16.8 weeks in the 12 week and 24 week groups respectively.

The mean on-treatment PEG-IFN adherence was similar between those receiving 12 and 24 weeks of therapy ( $98.5 \%$ vs. $98.7 \%, P=0.811$ ). The proportion of participants missing at least one dose of PEG-IFN while on therapy was $3 \%$ in those receiving 12 weeks of therapy

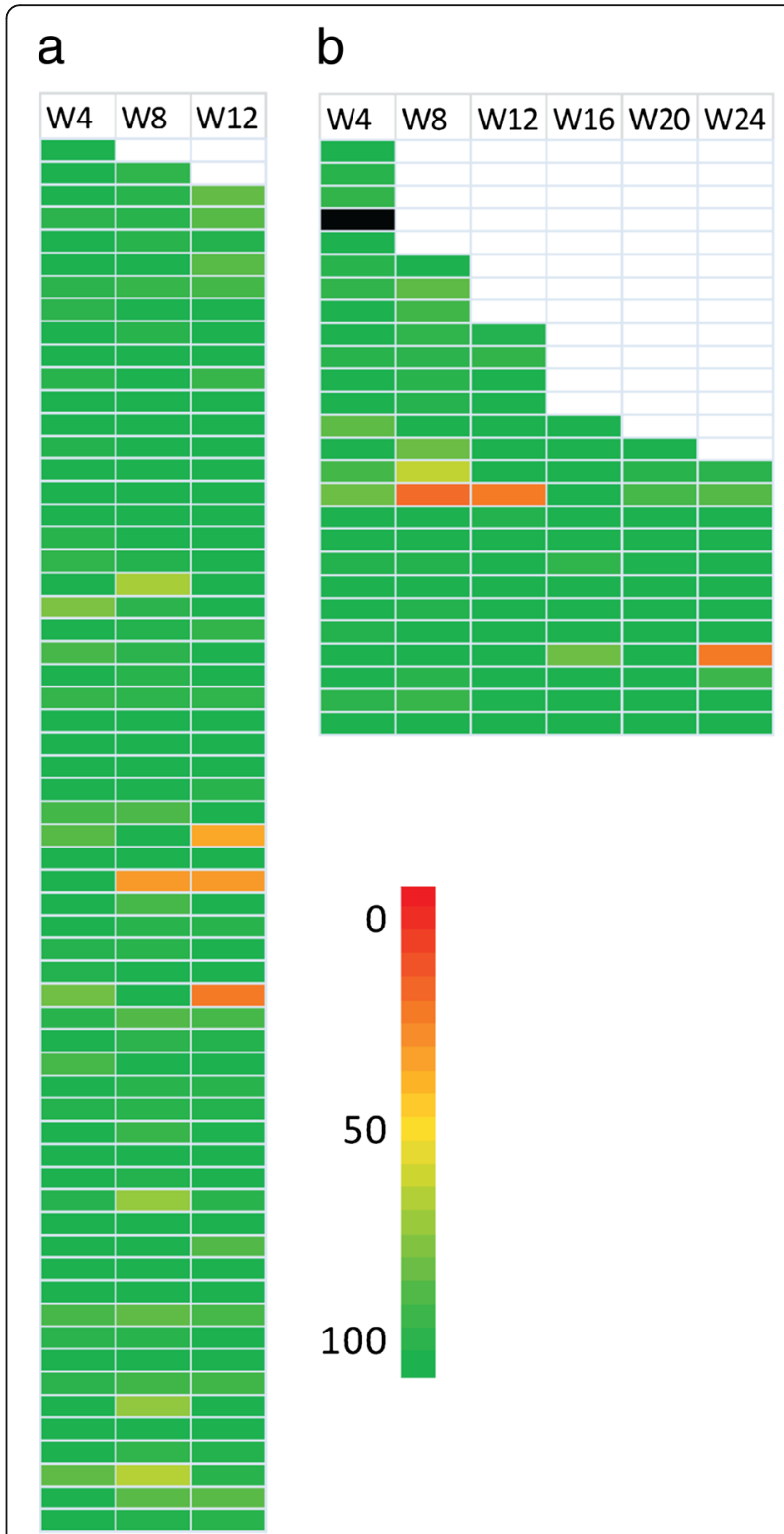

Fig. 3 Four-weekly RBV adherence among HCV genotype 2/3 participants on shortened (12 weeks; $n=61$; panel a) and standard therapy ( 24 weeks; $n=26$; panel b). Each row represents a study participant. Colours represent each participant's RBV adherence during each four-week period with dark green representing 100\% adherence and red representing $0 \%$ adherence. White represents discontinuation before completion of the corresponding time-point while black represents missing data for both retuned pill counts and patient reported adherence before treatment discontinuation 
Table 3 Unadjusted potential predictors of treatment completion among the study population $(n=93)$

\begin{tabular}{|c|c|c|c|c|c|}
\hline & $\begin{array}{l}\text { Number completed } \\
\text { treatment }(\% ; n=71\end{array}$ & $\begin{array}{l}\text { Number not completing } \\
\text { treatment }(\% ; n=22)\end{array}$ & Unadjusted OR & $95 \% \mathrm{Cl}$ & $P$ \\
\hline \multicolumn{6}{|l|}{ Age (years) } \\
\hline$\leq 41$ & $38(83)$ & $8(17)$ & 1.00 & - & - \\
\hline$>41$ & $33(70)$ & $14(30)$ & 0.50 & $0.19-1.33$ & 0.164 \\
\hline \multicolumn{6}{|l|}{ Gender } \\
\hline Female & $13(81)$ & $3(19)$ & 1.00 & - & - \\
\hline Male & $58(75)$ & $19(25)$ & 0.70 & $0.18-2.74$ & 0.613 \\
\hline \multicolumn{6}{|l|}{ Education $^{a}$} \\
\hline$<$ Tertiary & $52(79)$ & $14(21)$ & 1.00 & - & - \\
\hline Tertiary or greater & $18(75)$ & $6(25)$ & 0.81 & $0.27-2.42$ & 0.703 \\
\hline \multicolumn{6}{|c|}{ Social functioning score } \\
\hline$<17$ & $31(78)$ & $9(23)$ & 1.00 & - & - \\
\hline$\geq 17$ & $40(75)$ & $13(25)$ & 0.89 & $0.34-2.36$ & 0.820 \\
\hline \multicolumn{6}{|l|}{ Stable housing } \\
\hline No & $17(77)$ & $5(23)$ & 1.00 & - & - \\
\hline Yes & $54(76)$ & $17(24)$ & 0.93 & $0.30-2.91$ & 0.907 \\
\hline \multicolumn{6}{|l|}{ Current depression $^{b}$} \\
\hline No & $30(75)$ & $10(25)$ & 1.00 & - & - \\
\hline Yes & $37(80)$ & $9(20)$ & 1.37 & $0.49-3.80$ & 0.545 \\
\hline \multicolumn{6}{|l|}{$\begin{array}{l}\text { Hazardous alcohol } \\
\text { consumption }{ }^{c}\end{array}$} \\
\hline No & $58(81)$ & $14(19)$ & 1.00 & - & - \\
\hline Yes & $11(83)$ & $2(15)$ & 1.71 & $0.35-8.43$ & 0.512 \\
\hline \multicolumn{6}{|l|}{ Current OST } \\
\hline No & $19(70)$ & $8(30)$ & 1.00 & - & - \\
\hline Yes & $52(79)$ & $14(21)$ & 1.56 & $0.57-4.32$ & 0.388 \\
\hline \multicolumn{6}{|l|}{ Injecting (last month) } \\
\hline No & $26(68)$ & $12(32)$ & 1.00 & - & - \\
\hline Yes & $45(82)$ & $10(18)$ & 2.08 & $0.79-5.47$ & 0.139 \\
\hline \multicolumn{6}{|l|}{$\begin{array}{l}\text { Frequency of injecting } \\
\text { (last month) }\end{array}$} \\
\hline Never & $26(68)$ & $12(32)$ & 1.00 & - & - \\
\hline Less than weekly & $17(77)$ & $5(23)$ & 1.57 & $0.47-5.26$ & 0.465 \\
\hline Weekly or greater & $28(85)$ & $5(15)$ & 2.58 & $0.80-8.34$ & 0.112 \\
\hline \multicolumn{6}{|l|}{$\begin{array}{l}\text { Heroin injecting } \\
\text { (last month) }\end{array}$} \\
\hline No & $43(72)$ & $17(28)$ & 1.00 & - & - \\
\hline Yes & $28(85)$ & $5(15)$ & 2.21 & $0.73-6.68$ & 0.159 \\
\hline \multicolumn{6}{|l|}{$\begin{array}{l}\text { Cocaine injecting } \\
\text { (last month) }\end{array}$} \\
\hline No & $63(76)$ & $20(24)$ & 1.00 & - & - \\
\hline Yes & $8(80)$ & $2(20)$ & 1.27 & $0.25-6.48$ & 0.774 \\
\hline \multicolumn{6}{|c|}{$\begin{array}{l}\text { Amphetamine injecting } \\
\text { (last month) }\end{array}$} \\
\hline No & $63(80)$ & $16(20)$ & 1.00 & - & - \\
\hline Yes & $8(57)$ & $6(43)$ & 0.34 & $0.10-1.12$ & 0.075 \\
\hline
\end{tabular}


Table 3 Unadjusted potential predictors of treatment completion among the study population $(n=93)$ (Continued)

\begin{tabular}{|c|c|c|c|c|c|}
\hline \multicolumn{6}{|c|}{ Injecting on therapy } \\
\hline No & $30(83)$ & $6(17)$ & 1.00 & - & - \\
\hline Yes & $41(79)$ & $11(21)$ & 0.75 & $0.25-2.24$ & 0.601 \\
\hline \multicolumn{6}{|c|}{ Therapy duration* } \\
\hline 24 weeks & $13(50)$ & $13(50)$ & 1.00 & - & - \\
\hline 12 weeks & $58(95)$ & $3(5)$ & 19.33 & $4.81-77.78$ & $<0.001$ \\
\hline
\end{tabular}

compared to $15 \%$ in those receiving 24 weeks of therapy $(P=0.072)$.

Four-weekly RBV adherence among those receiving 12 weeks of therapy and 24 weeks of therapy is shown in Fig. 3. Returned pill counts were used in 276 of the 284 on treatment visits in Fig. 3 (97\%). In the case of missing pill counts $(n=8)$ participant completed questionnaire data was used where available $(n=7)$. Data was not available, either from pill counts or patient completed questionnaires for one study visit. The mean overall ontreatment RBV adherence was similar between those receiving 12 and 24 weeks of therapy (94.8\% vs. $94.1 \%$, $P=0.751)$. The mean on-treatment adherence to RBV in the 12 week arm during weeks $1-4,5-8$, and 9-12 was $96.9 \%, 93.8 \%$ and $93.5 \%$ respectively. The mean ontreatment adherence to RBV in the 24 week arm during weeks $1-4,5-8$, and $9-12$ was $96.6 \%, 88.3 \%$ and $95.2 \%$ respectively. Overall $65 \%$ of participants had $>95 \%$ ontreatment RBV adherence.

\section{Factors associated with treatment completion and on- treatment RBV adherence}

In unadjusted analyses, treatment completion occurred more frequently in those with recent injecting at baseline, recent heroin injecting at baseline, injecting weekly or more in the past month, and those in the 12 week arm but less often in people with recent amphetamine injecting at baseline and those who were $>41$ years of age (Table 3). The only factor that remained independently associated with treatment completion was shortened treatment arm (aOR 31.2, $P=<0.001$ ).

In unadjusted analyses, no factors were associated with $>95 \%$ on-treatment RBV adherence (Table 4). In GEE analyses, later study visit was not associated with adherence to RBV (OR 1.00, $P=0.489$ ).

\section{Impact of missed doses of PEG-IFN and RBV, and treat- ment completion on SVR}

In unadjusted analyses, SVR occurred more frequently among those who completed treatment (OR 34.4, $P=<0.001)$. Neither 100\% PEG-IFN adherence, nor $>95 \%$ RBV adherence were associated with SVR in unadjusted analyses and were therefore not included in the adjusted model. In adjusted analyses, when adjusted for all factors found to be associated with SVR in the primary analysis [11], treatment completion remained as being associated with SVR (aOR 23.86, $P=0.003$; Table 5).

\section{Discussion}

This study investigated treatment completion and the adherence to response-guided directly observed PEGIFN and self-administered ribavirin treatment for chronic HCV genotypes 2/3 among PWID with ongoing drug use and those receiving OST. The results demonstrated high adherence to directly observed PEG-IFN and self-administered RBV therapy, particularly among participants receiving 12 weeks, as opposed to 24 weeks, of therapy. Being scheduled to receive 12 weeks of therapy was an independent predictor of treatment completion. There were no independent predictors of $>95 \%$ RBV adherence in the population. Neither recent injection drug use prior to treatment, nor injection drug use while on treatment, were associated with treatment completion or $>95 \%$ on-treatment RBV adherence. Further, the majority of the sub-optimal treatment exposure was due to early discontinuation of therapy rather than missed doses while on therapy. Finally, while neither PEG-IFN nor RBV adherence was an independent predictor of SVR, treatment completion was found to be an independent predictor of SVR. These data have important clinical implications informing HCV management among PWID with ongoing drug use and those receiving OST in the DAA era, given that the majority of licensed regimens require only 12 weeks of therapy.

Overall, adherence to PEG-IFN/RBV therapy was high with few participants (6\%) missing $\geq 1$ dose of PEG-IFN while on therapy, with no one missing more than one dose for a high on-treatment adherence of $98 \%$. This is consistent with previous studies of PEG-IFN adherence where on-treatment adherence ranged from $74 \%$ to $99 \%$ [3, 14-17]. While slightly lower than PEG-IFN adherence, a similarly high on-treatment RBV adherence was observed $(94.6 \%)$ with $71 \%$ of participants taking at least $95 \%$ of their RBV doses. The proportion of the 
Table 4 Unadjusted potential predictors of RBV adherence among the study population with available RBV adherence data $(n=86)$

\begin{tabular}{|c|c|c|c|c|c|}
\hline & RBV adherence of 95\% $(\% ; n=59)$ & RBV adherence of $<95 \%(\% ; n=27)$ & Unadjusted OR & $95 \% \mathrm{Cl}$ & $P$ \\
\hline \multicolumn{6}{|l|}{ Age (years) } \\
\hline$\leq 41$ & $29(66)$ & $15(34)$ & 1.00 & - & - \\
\hline$>41$ & $30(71)$ & $12(29)$ & 1.25 & $0.50-3.11$ & 0.632 \\
\hline \multicolumn{6}{|l|}{ Gender } \\
\hline Female & $49(69)$ & $22(31)$ & 1.00 & - & - \\
\hline Male & $10(67)$ & $5(33)$ & 1.14 & $0.35-3.72$ & 0.833 \\
\hline \multicolumn{6}{|l|}{ Education $^{a}$} \\
\hline$<$ Tertiary & $43(69)$ & $19(31)$ & 1.00 & - & - \\
\hline Tertiary or greater & $15(71)$ & $6(29)$ & 1.18 & $0.40-3.48$ & 0.766 \\
\hline \multicolumn{6}{|l|}{ Social functioning score } \\
\hline$<17$ & $29(76)$ & $9(24)$ & 1.00 & - & - \\
\hline$\geq 17$ & $30(63)$ & $18(38)$ & 0.53 & $0.21-1.38$ & 0.195 \\
\hline \multicolumn{6}{|l|}{ Current depression $^{\mathrm{b}}$} \\
\hline No & $24(65)$ & $13(35)$ & 1.00 & - & - \\
\hline Yes & $30(68)$ & $14(32)$ & 1.11 & $0.44-2.80$ & 0.818 \\
\hline \multicolumn{6}{|c|}{ Hazardous alcohol consumption ${ }^{c}$} \\
\hline No & $48(68)$ & $23(32)$ & 1.00 & - & - \\
\hline Yes & $8(67)$ & $4(33)$ & 0.94 & $0.26-3.44$ & 0.924 \\
\hline \multicolumn{6}{|l|}{ Current OST } \\
\hline No & $16(64)$ & $9(36)$ & 1.00 & - & - \\
\hline Yes & $43(70)$ & $18(30)$ & 1.26 & $0.48-3.36$ & 0.638 \\
\hline \multicolumn{6}{|l|}{ Injecting (last month) } \\
\hline No & $22(69)$ & $10(31)$ & 1.00 & - & - \\
\hline Yes & $37(69)$ & $17(31)$ & 0.95 & $0.37-2.42$ & 0.908 \\
\hline \multicolumn{6}{|c|}{ Frequency of injecting (last month) } \\
\hline Never & $22(69)$ & $10(31)$ & 1.00 & - & - \\
\hline Less than weekly & $14(67)$ & $7(33)$ & 0.87 & $0.27-2.81$ & 0.815 \\
\hline Weekly or greater & $23(70)$ & $10(30)$ & 1.00 & $0.35-2.86$ & 1.000 \\
\hline \multicolumn{6}{|c|}{ Heroin injecting (last month) } \\
\hline No & $35(66)$ & $18(34)$ & 1.00 & - & - \\
\hline Yes & $24(73)$ & $9(27)$ & 1.33 & $0.51-3.46$ & 0.554 \\
\hline \multicolumn{6}{|c|}{ Cocaine injecting (last month) } \\
\hline No & $52(68)$ & $24(32)$ & 1.00 & - & - \\
\hline Yes & $7(70)$ & $3(30)$ & 0.77 & $0.25-4.44$ & 0.940 \\
\hline \multicolumn{6}{|c|}{ Amphetamine injecting (last month) } \\
\hline No & $51(69)$ & $23(31)$ & 1.00 & - & - \\
\hline Yes & $8(67)$ & $4(33)$ & 0.88 & $0.24-3.24$ & 0.853 \\
\hline \multicolumn{6}{|l|}{ Injecting on therapy } \\
\hline No & $25(74)$ & $9(26)$ & 1.00 & - & - \\
\hline Yes & $34(65)$ & $18(35)$ & 0.65 & $0.25-1.69$ & 0.380 \\
\hline \multicolumn{6}{|l|}{ Therapy duration } \\
\hline 24 weeks & $18(72)$ & $7(28)$ & 1.00 & - & - \\
\hline 12 weeks & $41(67)$ & $20(33)$ & 0.80 & $0.29-2.22$ & 0.664 \\
\hline
\end{tabular}


Table 5 Unadjusted and adjusted models of adherence-related predictors of SVR among the study population

\begin{tabular}{|c|c|c|c|c|c|c|c|}
\hline & SVR (\%) & Unadjusted OR & $95 \% \mathrm{Cl}$ & $P$ & Adjusted OR & $95 \% \mathrm{Cl}$ & $P$ \\
\hline \multicolumn{8}{|c|}{$95 \%$ ribavirin adherence* } \\
\hline No & $17(63)$ & 1.00 & - & - & - & - & - \\
\hline Yes & $44(73)$ & 1.62 & $0.61-4.26$ & 0.330 & - & - & \\
\hline \multicolumn{8}{|c|}{ 100\% PEG-IFN adherence* } \\
\hline No & $58(67)$ & 1.00 & - & - & - & - & - \\
\hline Yes & $3(50)$ & 2.00 & $0.38-10.53$ & 0.413 & - & - & - \\
\hline \multicolumn{8}{|c|}{ Completed therapy } \\
\hline No & $2(9)$ & 1.00 & - & - & 1.00 & - & - \\
\hline Yes & $59(83)$ & 34.41 & $6.90-171.55$ & 0.000 & 23.86 & $2.94-193.84$ & 0.003 \\
\hline
\end{tabular}

The adjusted model is adjusted for all factors associated with SVR from the primary analysis [11]

*On-treatment adherence

population with $>95 \%$ adherence is lower than was reported in the C-EDGE CO-STAR trial (96\%), a randomized controlled trial of elbasvir-grazoprevir in people receiving stable opioid agonist therapy [18]. This is potentially due to the higher toxicity of PEG-IFN/RBV therapy or the increased pill burden due to the inclusion of RBV. Also, the C-EDGE CO-STAR trial likely represents a more stable population given the inclusion of only those on stable OST and the exclusion of those actively using drugs of potential abuse. As such, it is difficult to directly compare these results.

Participants who were allocated 12 weeks of therapy demonstrated a higher proportion completing therapy than those allocated 24 weeks of therapy. Recent injection drug use prior to and during treatment was not associated with PEG-IFN adherence, RBV adherence, or treatment completion, consistent with previous data [3, 10, 19-22].

While PEG-IFN based therapies have recently been replaced by new DAA therapies in many settings, data on the adherence to HCV therapies among PWID is still needed. In many countries, concerns still exist regarding the adherence to therapy among active PWID $[10,23]$. As a result many countries have restricted the use of DAA therapies to those without current injecting [23-25]. A better understanding of adherence among active injectors is therefore needed to inform policy and remove the restrictions placed on DAA therapies. This data also highlights the positive effect of shortened therapy on treatment completion in this population as pushes to further shorten HCV therapy continue. This study includes data on adherence to self-administered ribavirin, providing some insight into adherence of an oral twice-daily antiviral therapy. Given the increased tolerability and simplicity DAA regimens compared to PEG-IFN and ribavirin-based therapies, adherence should be comparable, if not better, than that observed with twice-daily ribavirin, Further data is needed to assess adherence to DAA-based therapy among people with ongoing injecting drug use.
There are some limitations to this study. Adherence to RBV was determined based on returned pill counts where available and patient surveys where pill counts were unavailable. While pill counts are generally a better estimate of the true adherence as compared to patient reported adherence [26], there is still the potential for overestimation through lost pills. The measurement of adherence to PEG-IFN was more robust, given that this was a directly observed dose in the presence of the study nurse who recorded when the dose was taken. Further, adherence is a very complex phenomenon and may be influenced by a number of unmeasured factors (e.g. past experience with adherence to other medications, patient-doctor relationships). Lastly, the small sample size of this study is a limitation. With a larger sample size, it would be possible to more accurately estimate the true effects of various factors on HCV treatment adherence and the true effect of treatment adherence on SVR. In addition, while the international nature of this study increases the generalizability to globally diverse PWID populations, the participants recruited into this study may represent a somewhat selected group, based on improved engagement in care. Irrespective of these limitations, this is the first international study to evaluate adherence to HCV therapy among PWID and those receiving OST.

\section{Conclusions}

This study demonstrates high adherence to responseguided directly observed pegylated-interferon and selfadministered ribavirin therapy for chronic HCV genotypes $2 / 3$ infection among people with ongoing injecting drug use or receiving OST. These data suggest that shortening $\mathrm{HCV}$ therapy has the potential to increase treatment completion among PWID. This is of particular importance given the interest in evaluating shorter durations of DAA therapy. Further, injecting drug use both prior to and during treatment was not associated with reduced adherence to therapy or treatment completions. 
These data suggest that adherence to HCV therapy among HCV infected PWID is not compromised by ongoing injection drug use and supports guidelines which suggest that active PWID should not be excluded from therapy; rather decisions should be made on a case-bycase basis [27-30]. Further studies are needed to characterize adherence to interferon-free DAA therapies among PWID and those receiving OST to better understand whether adherence is actually a problem in this population in the DAA era.

\section{Additional file}

Additional file 1: Local ethics committees. (DOCX 16 kb)

\section{Abbreviations}

aOR: Adjusted odds ratio; DAA: Direct acting antiviral; HCV: Hepatitis C virus; HIV: Human immunodeficiency virus; OR: Odds ratio; OST: Opioid substitution therapy; PEG-IFN: Pegylated-interferon alfa-2b; PWID: People who inject drugs; RBV: Ribavirin; RVR: Rapid virological response; SVR: Sustained virological response

\section{Acknowledgements}

The authors would like to thank the study participants for their contribution to the research, as well as current and past researchers and staff. They would like to acknowledge members of the study group:

Protocol Steering Committee - Gregory Dore (Chair, UNSW Australia, Sydney, Australia), Jason Grebely (UNSW Australia, Sydney, Australia), Philip Bruggmann (Arud Centres for Addiction Medicine, Zurich, Switzerland), Philippa Marks (UNSW Australia, Sydney, Australia), Brian Conway (Vancouver Infectious Diseases Center, Vancouver, Canada), Geert Robaeys (Department of Gastroenterology and Hepatology, Ziekenhuis Oost Limburg, Genk, Belgium), Tracy Swan (Treatment Action Group, New York, United States), Graham Foster (The Liver Unit, Queen Mary University of London, London, United Kingdom), Markus Backmund (Ludwig Maximilians-University Munich, Munich, Germany) and Olav Dalgard (Akershus University Hospital, Oslo, Norway).

Coordinating Centre - Sophie Quiene (Study Co-ordinator), Evan Cunningham (PhD Student), Behzad Hajarizadeh (Associate Lecturer), Gregory Dore (Principal Investigator), Jason Grebely (Co-investigator), Pip Marks (Clinical Trials Manager), Ineke Shaw (Systems Manager), Sharmila Siriragavan (Data Manager) and Janaki Amin (Statistician).

Site Principal Investigators - Markus Backmund (PIT Munich, Munich, Germany), Stefan Bourgeois (ZNA Stuivenberg, Antwerp, Belgium), Philip Bruggmann (Arud Centres for Addiction Medicine, Zurich, Switzerland), Julie Bruneau (Centre Hôspitalier de l'Université de Montréal, Montréal, Canada), Brian Conway (Vancouver Infectious Diseases Center, Vancouver, Canada), Olav Dalgard (Akershus University Hospital, Oslo, Norway), Gregory Dore (St Vincent's Hospital, Sydney Australia), Adrian Dunlop (Newcastle Pharmacotherapy Service, Newcastle, Australia), Graham Foster (Tower Hamlets Specialist Addiction Unit, London, United Kingdom), Margaret Hellard (The Alfred Hospital, Melbourne, Australia), Jeff Powis (South Riverdale Community Health Centre, Toronto, Canada), Geert Robaeys (Department of Gastroenterology and Hepatology, Ziekenhuis Oost Limburg, Genk, Belgium), Stephen Ryder (Nottingham University Hospital, Nottingham, United Kingdom), Claude Scheidegger (Zentrum für Suchtmedizin, Basel, Switzerland), David Shaw (Royal Adelaide Hospital, Adelaide, Australia), Cornelia Staehelin (Poliklinik für Infektiologie, Inselspital, Bern, Switzerland) and Martin Weltman (Nepean Hospital, Penrith, Australia).

Site Co-ordinators - David Axten (Tower Hamlets Specialist Addiction Unit, London, United Kingdom), Jessica Andreassen and Ingunn Melkeraaen (Akershus University Hospital, Oslo, Norway), Anita Eevers (Department of Gastroenterology and Hepatology, Ziekenhuis Oost Limburg, Genk, Belgium), Catherine Ferguson (Royal Adelaide Hospital, Adelaide, Australia), Vincenzo Fragomeli (Nepean Hospital, Penrith, Australia), Susan Hazelwood and Rohan Holland (Newcastle Pharmacotherapy Service, Newcastle, Australia), Tina Horschik (Arud Centres for Addiction Medicine, Zurich, Switzerland), Christine
Huber (Zentrum für Suchtmedizin, Basel, Switzerland), Kate Jack (Nottingham University Hospital, Nottingham, United Kingdom), Barbara Kotsoros (Centre Hôspitalier de l'Université de Montréal, Montréal, Canada), Melanie Lacalamita (Poliklinik für Infektiologie, Inselspital, Bern, Switzerland), Kristof Lesneuck (ZNA Stuivenberg, Antwerp, Belgium), Kate Mason (South Riverdale Community Health Centre, Toronto, Canada), Alison Sevehon (St Vincent's Hospital, Sydney, Australia), Shawn Sharma (Vancouver Infectious Diseases Center, Vancouver, Canada), Sally Von Bibra (The Alfred Hospital, Melbourne, Australia), Nicole Widder (PIT Munich, Munich, Germany).

\section{Funding}

Supported in part by a research grant from the Investigator Initiated Studies Program of Merck Sharp \& Dohme Corp. The opinions expressed in this paper are those of the authors and do not necessarily represent those of Merck Sharp \& Dohme Corp. The Kirby Institute is funded by the Australian Government Department of Health and Ageing. The views expressed in this publication do not necessarily represent the position of the Australian Government. JG is supported by a National Health and Medical Research Council Career Development Fellowship. BH is supported by a National Health and Medical Research Council Early Career Fellowship. MH is supported by a National Health and Medical Research Council Principal Research Fellowship. GD is supported by a National Health and Medical Research Council Practitioner Research Fellowships.

\section{Availability of data and materials}

The data in this study are available upon request. The data cannot be publicly available because they contain confidential and potentially identifying information. Data will be made available to all interested researchers upon request. Data requests should be submitted to ACTIVATE Steering Committee (Professor Greg Dore, the chair: gdore@kirby.unsw.edu.au).

\section{Authors' contributions}

$\mathrm{OD}, J \mathrm{~A}, \mathrm{MH}, \mathrm{GRF}, \mathrm{PB}, \mathrm{BC}, \mathrm{MB}, \mathrm{GR}$, TS, PSM, SQ, TLA, MW, DS, AD, JB, SB, MCT, GJD, JG were responsible for design and implementation of the study. JG and $E B C$ were responsible for conception of this analysis. EBC, JG, JA, BH, HM were responsible for analysis of the data. EBC was responsible for writing the manuscript. All authors reviewed the manuscript and gave approval for publication.

\section{Competing interests}

JG is a consultant/advisor and has received research grants from Abbvie, Bristol Myers Squibb, Cepheid, Gilead Sciences and Merck/MSD and is an associate editor for BMC Infectious Diseases. OD is a consultant/advisor and has received research grants from Abbvie, Gilead Sciences and Merck/MSD. GRF is a consultant/advisor and has received research grants from Abbvie, Bristol Myers Squibb, Gilead Sciences, Janssen, Roche and Merck/MSD. PB is consultant/advisor and has received research grants from Abbvie, Bristol Myers Squibb, Gilead, Janssen and Merck. BC is consultant/advisor and has received research grants from AbbVie, Gilead and Merck. GR has received research grants from Merck Sharp \& Dohme, AbbVie, Janssen

Pharmaceuticals, and has acted as a consultant/advisor and for Gilead Sciences, Abbvie, Merck Sharp \& Dohme, and Bristol Myers-Squibb. MW is a consultant/advisor for Abbvie, Bristol Myers Squibb, Gilead, Merck, Janssen, Novartis and Roche. MH has received funding from Gilead Sciences, Abbvie and Bristol Myers Squibb for investigator initiated independent research. JB is a consultant/advisor and has received research grants from Gilead Sciences and Merck/MSD. SB is a consultant/advisor and has received research grants from AbbVie, MSD, BMS, Gilead, Janssen. CS has received travel grants through her institution sponsored by Gilead Sciences, Janssen and Merck/ MSD. GD is a consultant/advisor and has received research grants from Abbvie, Bristol Myers Squibb, Gilead, Merck, Janssen and Roche.

\section{Ethics approval and consent to participate}

All study participants provided written informed consent before study procedures. The study protocol was approved by St. Vincent's Hospital, Sydney Human Research Ethics Committee (primary study committee), as well as through local ethics committees (Additional file 1) at all study sites, and was conducted according to the Declaration of Helsinki and International Conference on Harmonization Good Clinical Practice (ICH/GCP) guidelines. The study was registered with clinicaltrials.gov registry 
(NCT01364090). The sponsor (The Kirby Institute, UNSW Australia) collected the data, monitored study conduct and performed the statistical analysis. An independant data and safety monitoring board reviewed the progress of the study.

\section{Consent for publication}

Not applicable.

\section{Publisher's Note}

Springer Nature remains neutral with regard to jurisdictional claims in published maps and institutional affiliations.

\begin{abstract}
Author details
${ }^{1}$ The Kirby Institute, UNSW Sydney, Sydney, NSW, Australia. ${ }^{2}$ Akershus University Hospital, Oslo, Norway. ${ }^{3}$ The Liver Unit, Queen Mary University of London, London, UK. ${ }^{4}$ Arud Centres for Addiction Medicine, Zurich, Switzerland. ${ }^{5}$ Vancouver Infectious Diseases Center, Vancouver, BC, Canada. ${ }^{6}$ Ludwig Maximilians-University Munich, Munich, Germany. ${ }^{7}$ Department of Gastroenterology and Hepatology, Ziekenhuis Oost Limburg, Genk, Belgium ${ }^{8}$ Department of Hepatology, UZ Leuven, Leuven, Belgium. ${ }^{9}$ UHasselt, Hasselt, Belgium. ${ }^{10}$ Treatment Action Group, New York, NY, USA. ${ }^{11}$ Nepean Hospital, Sydney, NSW, Australia. ${ }^{12}$ Royal Adelaide Hospital, Adelaide, South Australia, Australia. ${ }^{13}$ School of Medicine and Public Health, University of Newcastle, Newcastle, NSW, Australia. ${ }^{14}$ Burnet Institute, Melbourne, VIC, Australia. ${ }^{15}$ Research Center, Centre Hospitalier de I'Universite de Montreal (CRCHUM), Montreal, Quebec, Canada. ${ }^{16}$ Stuivenberg ZNA, Antwerp, Belgium.

${ }^{17}$ Department of Infectious Diseases, Bern University Hospital, University of Bern, Bern, Switzerland.
\end{abstract}

\section{Received: 11 January 2017 Accepted: 1 June 2017}

\section{Published online: 13 June 2017}

\section{References}

1. Nelson PK, Mathers BM, Cowie B, Hagan H, Des Jarlais D, Horyniak D, et al. Global epidemiology of hepatitis B and hepatitis $C$ in people who inject drugs: results of systematic reviews. Lancet. 2011;378(9791):571-83.

2. Hajarizadeh B, Grebely J, Dore GJ. Epidemiology and natural history of HCV infection. Nat Rev Gastroenterol Hepatol. 2013;10(9):553-62.

3. Grebely J, Matthews GV, Hellard M, Shaw D, van Beek I, Petoumenos K, et al. Adherence to treatment for recently acquired hepatitis $\mathrm{C}$ virus ( $\mathrm{HCV}$ ) infection among injecting drug users. J Hepatol. 2011:55(1):76-85.

4. Sylvestre $\mathrm{DL}$, Clements $\mathrm{BJ}$. Adherence to hepatitis $C$ treatment in recovering heroin users maintained on methadone. Eur J Gastroenterol Hepatol. 2007; 19(9):741-7.

5. Manolakopoulos S, Deutsch MJ, Anagnostou O, Karatapanis S, Tiniakou E, Papatheodoridis GV, et al. Substitution treatment or active intravenous drug use should not be contraindications for antiviral treatment in drug users with chronic hepatitis C. Liver Int. 2010;30(10):1454-60.

6. Sasadeusz JJ, Dore G, Kronborg I, Barton D, Yoshihara M, Weltman M. Clinical experience with the treatment of hepatitis $C$ infection in patients on opioid pharmacotherapy. Addiction. 2011;106(5):977-84.

7. Weiss JJ, Brau N, Stivala A, Swan T, Fishbein D. Review article: adherence to medication for chronic hepatitis $C$ - building on the model of human immunodeficiency virus antiretroviral adherence research. Aliment Pharmacol Ther. 2009:30(1):14-27.

8. Burkhart PV, Sabate E. Adherence to long-term therapies: evidence for action. J Nurs Scholarship. 2003;35(3):207.

9. Cramer JA, Roy A, Burrell A, Fairchild CJ, Fuldeore MJ, Ollendorf DA, et al. Medication compliance and persistence: terminology and definitions. Value Health. 2008;11(1):44-7.

10. Grebely J, Tyndall MW. Management of HCV and HIV infections among people who inject drugs. Curr Opin HIV AIDS. 2011;6(6):501-7.

11. Grebely J, Dalgard O, Cunningham EB, Hajarizadeh B, Foster GR, Bruggmann $P$. Efficacy of response-guided directly observed pegylated interferon and self-administered ribavirin for people who inject drugs with hepatitis $C$ virus genotype 2/3 infection: the ACTIVATE study. IJDP. 2016. In Press.

12. Maher L, Li J, Jalaludin B, Chant KG, Kaldor JM. High hepatitis $C$ incidence in new injecting drug users: a policy failure? Aust N Z J Public Health. 2007; 31(1):30-5.

13. Bush K, Kivlahan DR, McDonell MB, Fihn SD, Bradley KA. The AUDIT alcohol consumption questions (AUDIT-C): an effective brief screening test for problem drinking. Ambulatory care quality improvement project (ACQUIP). Alcohol use disorders identification test. Arch Intern Med. 1998;158(16):1789-95

14. Fumaz CR, Muñoz-Moreno JA, Ballesteros AL, Paredes R, Ferrer MJ, Salas A, et al. Influence of the type of pegylated interferon on the onset of depressive and neuropsychiatric symptoms in HIV-HCV coinfected patients. AIDS Care. 2007;19(1):138-45.

15. Smith SR, Wahed AS, Kelley SS, Conjeevaram HS, Robuck PR, Fried MW. Assessing the validity of self-reported medication adherence in hepatitis $C$ treatment. Ann Pharmacother. 2007;41(7):1116-23.

16. Weiss JJ, Bhatti L, Dieterich DT, Edlin BR, Fishbein DA, Goetz MB, et al. Hepatitis C patients' self-reported adherence to treatment with pegylated interferon and ribavirin. Aliment Pharmacol Ther 2008:28(3):289-93.

17. Cacoub P, Melin P, Ouzan D, Cattan L, Chousterman M, Varastet M, et al. 776 patient education improves adherence to PEGINTERFERON and ribavirin in chronic genotype 2 or 3 hepatitis c virus (HCV) infection in the real-Life. J Hepatol. 2008;48:S289-90.

18. Dore G, Altice F, Litwin AH, Dalgard O, Gane EJ, Shibolet O, Luetkemeyer A, Nahass R, Peng CY, Conway B et al:C-EDGE CO-STAR: Efficacy of Grazoprevir and Elbasvir in Persons who Inject Drugs (PWID) Receiving Opioid Agonist Therapy.Hepatology 2015, 62:227a-228a.

19. Marcellin P, Chousterman M, Fontanges T, Ouzan D, Rotily M, Varastet M, et al. Adherence to treatment and quality of life during hepatitis $C$ therapy: a prospective, real-life, observational study. Liver Int. 2011;31(4):516-24.

20. Manolakopoulos S, Deutsch MJ, Anagnostou O, Karatapanis S, Tiniakou E, Papatheodoridis GV, et al. Substitution treatment or active intravenous drug use should not be contraindications for antiviral treatment in drug users with chronic hepatitis C. Liver Int. 2010;30(10):1454-60.

21. Wilkinson M, Crawford V, Tippet A, Jolly F, Turton J, Sims E, et al. Community-based treatment for chronic hepatitis $C$ in drug users: high rates of compliance with therapy despite ongoing drug use. Aliment Pharmacol Ther. 2009;29(1):29-37.

22. Sylvestre $\mathrm{DL}$, Clements BJ. Adherence to hepatitis $C$ treatment in recovering heroin users maintained on methadone. Eur J Gastroenterol Hepatol. 2007;19(9):741-7.

23. Marshall AD, Nielsen S, Cunningham EB, Aghemo A, Alho H, Backmund M, Bruggmann P, Dalgard O: Restrictions for reimbursement of interferon-free direct acting antiviral therapies for HCV infection in Europe. ILC 2017 - LBP5052017.

24. Barua S, Greenwald R, Grebely J, Dore GJ, Swan T, Taylor LE. Restrictions for Medicaid Reimbursement of Sofosbuvir for the Treatment of Hepatitis C Virus Infection in the United States. Annals of Internal Medicine. 2015;163(3):215.

25. Marshall AD, Saeed S, Barrett L, Cooper CL, Treloar C, Bruneau J, et al. Restrictions for reimbursement of direct-acting antiviral treatment for hepatitis C virus infection in Canada: a descriptive study. CMAJ open. 2016; 4(4):E605-14.

26. Weiss JJ, BrÄU N, Stivala A, Swan T, Fishbein D. Review article: adherence to medication for chronic hepatitis $C$ - building on the model of human immunodeficiency virus antiretroviral adherence research. Aliment Pharmacol Ther. 2009:30(1):14-27.

27. Swan T, Curry J. Comment on the updated AASLD practice guidelines for the diagnosis, management, and treatment of hepatitis $\mathrm{C}$ : treating active drug users. Hepatology. 2009;50(1):323-4.

28. Ghany MG, Strader DB, Thomas DL, Seeff LB. Diagnosis, management, and treatment of hepatitis C: an update. Hepatology. 2008;49(4):1335-74.

29. Grebely J, Haire B, Taylor LE, Macneill P, Litwin AH, Swan T, et al. Excluding people who use drugs or alcohol from access to hepatitis $C$ treatments - is this fair, given the available data? J Hepatol. 2015;63(4):779-82.

30. Grebely J, Robaeys G, Bruggmann P, Aghemo A, Backmund M, Bruneau J, et al. Recommendations for the management of hepatitis $C$ virus infection among people who inject drugs. The International journal on drug policy. 2015;26(10):1028-38. 